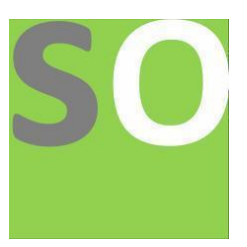

Article title: Ineffective management practices on infection prevention and control by nurses in a public hospital Authors: Thizwilondi Magadze[1], Dayanithee Chetty[2], Enneth Nkhwashu[3]

Affiliations: Sefako Makgatho University[1]

Orcid ids: 0000-0001-5394-6447[1]

Contact e-mail: ananias.magadze@gauteng.gov.za

License information: This work has been published open access under Creative Commons Attribution License http://creativecommons.org/licenses/by/4.0/, which permits unrestricted use, distribution, and reproduction in any medium, provided the original work is properly cited. Conditions, terms of use and publishing policy can be found at https://www.scienceopen.com/.

Preprint statement: This article is a preprint and has not been peer-reviewed, under consideration and submitted to ScienceOpen Preprints for open peer review.

Funder: none

DOI: 10.14293/S2199-1006.1.SOR-.PPUYY0R.v1

Preprint first posted online: 18 January 2022

Keywords: Infection prevention and control measures, ineffective management practices, Hospital Acquired infections 


\title{
Title: Ineffective management practices on infection prevention and control by nurses in a public hospital.
}

\section{Thizwilondi Ananias Magadze}

Department of Nursing Science, Sefako Makgatho Health Sciences University, Ga-Rankuwa, Republic of South Africa (+27726436543) ORCID ID: https://Orcid.org/00000001-5394-6447

\begin{abstract}
This research aims to explore the ineffective management practices on infection prevention and control by nurses in a public hospital. This was done through focus groups with nurses from different public hospitals in Tshwane area. Participants expressed that there are ineffective management practices in their working environment in the form of inadequate education, training on infection and control measures, shortage of human and material resources and poor communication that is affecting the implementation of infection prevention and control measures in the hospital. Results: Most nurses have been found to have attended an hour to a day IPC training, which they alluded has no impact to their implementation as is too short. Study also found that institutions where nursing qualifications was obtained has significant impact to implementation of IPC measures. There was an agreement that continuous training on IPC related issues improve in practice and confidence to nurses

Study also found that the presence of well-trained nurse is vital in the ward, however the ward has to meet other aspects such as staffing, skill mix, resources and working conditions. Best practices that also involve behaviour changes were found to be successful in intervention to improve implementation of IPC measure. Ongoing feedback and full training on IPC were found significant to improve IPC measures.
\end{abstract}

Key words: Infection prevention and control measures, ineffective management practices, Hospital Acquired infections

\section{Introduction}

The significance of preventing and controlling infections, dates back from 1854, from the days of Florence Nightingale who observed and advocated that improving hygienic conditions, through a clean environment, maintaining patient cleanliness and hand hygiene decrease the number of infection- related patients' deaths. Such line of thinking resulted in the emergence of a nurse primarily dedicated towards infection control duties

Currently, this nurse is known as an IPC Practitioner, with the roles and functions of educating, intervening and evaluating the practice of preventing and controlling infections with patient care being the primary focus (Lewinsky, 2011: 776). However, such changes 
have yielded very few results as currently very few universities and school offer the formal training for such nurses. Colleges and Universities offer the course as specialty despite that is needed by all nurses in the hospitals.

An observational study conducted by Brouwer, Coelho, Das Dores Mosse, Brondi, Winterton and Van Leth in Mozambique (2014:5-10) found that despite the policy on IPC management being available in the hospital. Very few recommendations were found to be applied in practice, and many nurses had a poor knowledge of the related policies and its practices.

Ider, Adams, Marton, Whitby and Clement (2012: 174-175) study done in Mongolia highlights that the compliance of hand hygiene, the attitude and knowledge among the nursing staff, and the biggest challenge in non-adherence to infection control measures were the doctors were in the public hospital. Gammon (2008:157-167) in United Kingdom (UK), supported that there was sub-optimal compliance to the IPC program in the public hospitals, evidenced by poor IPC measures, such as hand washing, isolation and aseptic techniques during wound dressing varied amongst the healthcare workers, despite the available resources and the knowledge of the nurses.

Systematic reviews of literature conducted by Ider, Adam, Marton, Whitby and Clemets (2012: 175) identified that both developed and developing countries have high numbers of cases of the HCAl problem. Ider et al (2012: 175) informed that HCAl was the most frequent adverse event in health care, was the biggest challenge around the world, and remains unknown due to the difficulty in obtaining reliable data.

\section{Aim of study}

to explore and develop guidelines that will improve the implementation of the infection prevention and control measures in the public hospitals

\section{Objective}

To explore the management practices on IPC measures in a public hospital

\section{Research and Methodology}


A qualitative, exploratory, descriptive and contextual research design was used. The approach and designs enabled the researchers to gain more information regarding the nurses' experiences in the management of IPC measure in a public hospital. Each hospital was coded as Academic hospital (AHS00), Regional hospital (RHS04) and District hospital (DHS08). The study was conducted within the nurses practice areas. Focus group interviews and field notes were used to collect data. A voice-recorder device was used as per participant's permission for the researchers to capture and preserve the data as accurately as possible (Creswell 2013, 244).

The participants were informed of their rights to make their own decision voluntarily to participate in the research. Sample of focus group and sample size illustrated below:

\begin{tabular}{|l|l|}
\hline Focus group & Sample size $(\mathrm{N})$ \\
\hline Nurses from 2 Academic hospitals & \\
-Academic Hospital 1 & 10 \\
-Academic Hospital 2 & 9 \\
\hline Nurses from Regional hospital & 10 \\
\hline Nurses from 2 District hospitals & 10 \\
-District hospital 1 & 10 \\
-district hospital 2 & Total participants $(\mathrm{N})=49$ \\
\hline Total focus groups $(\mathrm{N})=5$ & - 59 \\
\hline
\end{tabular}

Five focus groups comprised of all nurses working directly with the patients in academic hospital (ahs00), regional hospital (rhs04) and district hospital (dhs08) gave their views regarding their experiences on the factor that affect the implementation of infection prevention and control measures public hospitals.

The researcher and his assistant team arrived at different hospitals as arrangement with supervisors. The boardrooms were ear marked in all hospitals, however, in one district hospital; there were changes due to urgent meeting, and meeting was re directed to nurse managers office.

\section{Ethical Consideration}

The study was approved by the ethics committee of the Sefako Makgatho Health Sciences University (SMUREC/H/228/2018:PG). Permission to conduct the research was obtained from the different health authorities to ensure ethical compliance of the research 
and for approval. For research to be considered ethical, the rights of the participants are of paramount importance. According to Grove et al. $(2013,162)$, the ethical research principles were formulated to protect the human rights of the participants, namely: the right to self-determination, right to privacy, right to anonymity and confidentiality, right to fair treatment and right to protection from discomfort and harm. Informed consent was obtained from each participant before undertaking the research, so that all were well informed about the benefits and risks of the research. They were made aware that they were free to withdraw from an interview at any time without penalty.

\section{Result and Discussion}

The participants expressed that there are ineffective management practices in their working environment, whereby three subthemes were identified in the form of inadequate education, training on infection and control measures, shortage of human and material resources and poor communication that is affecting the implementation of infection prevention and control measures in the hospital...

Ider et al (2012:8) confirmed that ineffective manager is not proactive but respond to situations associated with implementation of infection and control measures when they have occurred The above quotes were supported by Efstathiou; Papastavrou; Raftopoulos and Merkouris (2011: 1) who asserted that the existence of an ineffective management practice, deters management from supporting the nurses with the resolving of issues experienced at ground level.one participant say

"My Manager demands us to comply with IPC policies that she does not understand".

\section{THEME ONE: Lack of adequate education and training on infection prevention and control measures}

Participants in this study identified lack of adequate education and training as influential to the application of infection prevention and control measures, as evidenced by the following direct quotations from the participants. Amoran and Onwube (2013:156-163) study found that nurse who have been trained on infection control principles are likely to implement the measures than those that are not trained. 
Clynes; Hourican; Kicullen; Lawrence; MacDermatton; O'Neill; Raftery and Stan (2010: 68) who found that nurses can minimize the incidence of hospital infections if they operate towards standard operation procedures in infection control. They also highlighted that the role of nurses in IPC tends to be underplayed because of their different knowledge and levels in healthcare facilities.

Participant was quoted saying; "I never got any training from the school I attended on these things. We just do prevention during procedures in OSCE" Neglect of employees training is mentioned by the National Infection Prevention and Control Policy and Strategy, NDoH (a) (2007:30-31) that underscored the need for nurses to be trained on infection prevention and control measures as a way of reducing infections during patient care.

All participants believe that having such training will reduce non-compliance. The following quotations were evident:

"But, if we are getting training from schools, the knowledge will be beneficial to us than be taught many policies as new during employment. I agree that we can perform better than this if training was offered at that time"

\section{THEME TWO. Inadequate Human and material resources}

Participants indicated that they are having shortage of resources that influences the implementation of infection prevention and control. Such resources like hand washing soap, hand rub and hand paper towel, were asserted as very critical to minimise risk of infections without working materials.

Participant quoted saying: "this hospital, management does not care, there is nothing, I mean no resources for infection prevention and control in the institution"

In this study, material resources will mean resources related to the management of infection prevention and control measures such as alcohol hand rub solutions, gloves, gowns, hand paper towel, and personal protective equipment The participants asserted the lack of material resources as a factor that influences the implementation of infection prevention and control measures. 
Material resources were reported to influence the implementation of infection prevention and control measures, however most healthcare facilities were found not prioritising such important issues (Ogoina et al 2015: 16-22)

Vukoja, Riviello, Schultz (2018:421-427) highlighted that, the wrong type materials of PPE were reportedly found in most areas of clinical practices while used by nurse. This has been found compromising the prevention and control of infection, despite that the several attempts were made for management to be aware

Participants said; "...everything is out of stock in this hospital”

Unavailability of protective equipment's was seen as influence on personal protective equipment (PPE) non-adherence in different working areas

Participants claimed that poor procurement processes negatively affected the implementation of infection prevention and control measures.

Shortage of staff has devastating consequences in patient care. They suggested that issues of staff must be taken into consideration for effective infection prevention and control management (Gammon et al 2008: 157-167). Participants indicated that shortages in human resources were negatively affecting the implementation of infection prevention and control measures. One participant was recorded saying; "It's difficult to implement some measures when we are short staffing"

\section{THEME THREE: Ineffective communication and support}

Markman (2017) who regards ineffective communication as miscommunication attributable to differences in languages and other factors indicates that effective communication can only be accomplished when there is an atmosphere that is characterised by mutual respect, support and confidence. Ineffective communication refers to the breakdown in communication between two parties, particularly when both fail to deliver messages or process received messages (AI Shamsi et al., 2020:122).

In this regard, Al Shamsi et al (2020:122) stressed that the problem of ineffective communication is caused by obstacles such as attitude, lack of knowledge of subject, assumptions, language barrier and structural problems in organisations.

A participant was quoted saying: "We are not formerly informed on what is happening with regard to infection prevention and control policies." 


\section{Lack of support}

Birgand, Johansson, Szilagyi, and Lucet (2015:1067-1071) study also observed that lack of support was associated with failure to attain infection prevention and control objectives. Investigations into infection outbreaks have illustrated that infection control teams encounter difficulties that cut across operational units and management level due to lack of support.

IPC Policy and Strategy NDOH (a) (2007:33) addresses the lack of support by management on infection prevention and control issues and emphasises the need for management buy in and support for the nurses to improve the implementation of infection prevention and control measures.

\section{Limitations of the Study}

The researchers identified the following restrictions that may affect the credibility and generalisability of the findings of this study:

As the study was conducted in one province and district (Gauteng at Tshwane district), it is entirely possible that the findings would differ if the same study were to be conducted in another province cannot be disputed.

Accessing the participants during guidelines development was very difficult due to restriction of movement due to Covid 19 outbreak. This might have different outcome if the team has set down and discuss that exchange through telephone and emails, as this need network data. Talking has proved to be more effective than writing as the participant is able to express freely.

Collection of the data was difficult as it was not possible to get all nurses together due to the off-duty schedule and unavailability of some participants during qualitative data collection

Data collection was done in three public hospitals; however sufficient data collected proved to be sufficient to answer the research question and to address the objectives of this study.

The researcher works in one of the public hospitals where the study was conducted.

This could have affected participation by some potential participants; however, bracketing was done to establish trustworthiness of the study 


\section{Recommendations}

- Management must support and listen the employees on issues related to IPC Measures

- Management must involve the employee through active participation during planning.

-Management must participate actively in IPC measures by monitoring and ensuring adherence

-Management is mandated to implement measures outlined in the National Core Standards guideline

-such as cleanliness, infection prevention and control, Availability of resources and attitude management.

-Management must ensure supervision regarding IPC measures by applying effective management practices such as good communication, leading by example, effective delegation and staff development

-Management must provide meaningful feedback to the staff to keep the staff abreast with information, as to build trust

-All levels of management must receive adequate training on all management skills

-Management must be a role model to the staff so that positive way is displayed.

-Management must improve on employee recognition.

-Nurse managers must Lead by example towards common goal

-Nursing unit and department must set communication standards to prevent miscommunication

-Nurse manager must encourage nurses to raise all communication challenges to forge working relationship to find solution

-Managers for nursing must stay consistent with expectations and follow up

-Managers must offer constructive feedback and complaints, while nurses seek feedback and participation regularly

-Managers must clearly define the nursing roles and responsibilities

-Culture of respect must be created

-All nursing workflows must be kept transparent

In the development of personal and professional leadership and infection control management skills of nurses and student nurses, NEls preceptors together with clinical preceptor nurses should:

- Attend periodic refresher courses, on-the-job training and supervised practice to ensure competent and adequate performance. 
- Conduct on-site training and periodic training updates to fill the identified gaps in provider knowledge, skills, values and attitudes.

- Conduct structured orientation sessions for new staff and nursing students, as well as regular sessions on new developments senior nurses.

- Develop infection control management skills training programmes that include quality improvement methods, surveillance system, isolation and collection and collation of patient data.

- Ensure that preceptors for nurses are always available in facilities to provide structured supportive supervision and monitoring to nurses and students, in addition to the provision of patient care.

- Establish formal recognition programmes, such as "employee of the month", to strengthen good performance.

- Familiarise nurses and student nurses with institutional enabling standard operating procedures which are aligned to provincial and national enabling prescripts for the provision of contraceptive services.

- Integrate leadership and management of infections content, including advocacy and professionalism, in the curriculum for nursing students.

- Regularly provide updated training for academic staff and managers/supervisors to enable them to give support and mentor nurses on all aspects of infection control.

- Train nurse managers, IPC practitioners and operational managers on coaching and facilitation skills.

In the development of nurses and student nurses' competencies on management of IPC stock, nurses learning packages should contain knowledge and skills on:

- Appropriate storage procedures and quality standards for equipment and suppliers of IPC.

- Creation of databases on quantity to be ordered based on past use and previous statistics of infections

- Development of institutional policies to deal with emergency orders, expired equipment 'sand borrowing of supplies from other facilities if there is a sudden increase in demand due to outbreaks.

- Establishment of efficient systems for inventory control, projection of supply needs, procurement and distribution within the scope of practice of nurse within the hospital with the support of supply chain unit. 
- Implementation of record-keeping systems to ensure replenishment of stocks, including ensuring availability of cleaning resources.

- Implementation of the procedures and logistics of obtaining IPC stocks, including orientation on national standards for the equipment and suppliers necessary to deliver high-quality personal protective equipment's.

- National, provincial and local policies and standard operating procedures on efficient management of IPC stock.

- Stock ordering and stock procedures for the unit which are aligned to the national, provincial and hospital IPC guidelines. This should include inventory and reordering protocols.

To harmonise and influence involvement of employee unions in daily nursing practices:

- Nurse managers and employee unions can also work together in teams to influence nurses to improve nursing practices, to enhance their working relationship.

- Nursing managers must improve their relationships with employee unions and nurses through the adoption of proactive employment policies.

- Regular communication between the nurse managers and the nurses improves the quality of the working relationship and minimizes conflicts

- Steps should be taken to train nurses in the use of communication methods and to make them as far as possible conversant with all the subjects in respect of which communication takes place. Training for nurses' representative should therefore be promoted and given when needed.

- the nurse managers should ensure that information is given, and that consultation takes place between the parties such as employee unions concerned before decisions on matters of major interest are taken.

It is recommended that:

- Refresher course on IPC measures to be developed and implemented for nurses

- Introduction of IPC training as a module within the institutions for all nursing staff to attend

- Training of the management on IPC measures

- Education and training that clearly outlines the roles and task of nurses on infection prevention and control measures

- Infection prevention and control should have a full module in the undergraduate and post graduate curriculum in nursing education institutes 


\section{Conclusions}

The study found out that the infection prevention and control measures were not adequately adhered to in all public hospitals that were sampled in Tshwane District.

Meanwhile the patients, healthcare workers and community need protection from dangers of infections that emanate from ignorance of IPC measures as well as the provision of resources for personal health, which can be achieved through good IPC practices. Extensive training and education modification, resources, and improved management were found to be the solution of the gap in which quality patient care can be improved.

\section{References}

Al Shamsi, H.; Almutairi, A. G.; Al Mashrafi, S., and Al Kalbani, T. (2020). Implications of Language Barriers for Healthcare: A Systematic Review. Oman medical journal, Vol.35(2): e122

Amoran, O. and Onwube, O. (2013). Infection Control and Practice of Standard Precautions among Healthcare Workers in Northern Nigeria. Journal of Global Infectious Disease. Vol. 5 (4):156-163

Birgand G; Johansson A; Szilagyi E, and Lucet J.C (2015). Overcoming the obstacles of implementing infection prevention and control guidelines. Clin Microbiol Infect. Vol.21(12):1067-1071.

Brouwer, M.; Coelho E.; Das Dores Mosse, C.; Brondi, L.; Winterton, L. and Van Leth, F. (2014). Healthcare Workers' Challenges in the Implementation of Tuberculosis Infection Prevention and Control Measures in Mozambique. Tuberculosis infection prevention and control Mozambique. PLoS One. Open access Journal. Vol. 9(12): 1-12.

Chen, Y.; Liu, C. and Hwang, H. (2011). Key factors affecting healthcare professionals to adopt knowledge management: The case of infection control departments of Taiwanese hospitals. Experts System with Application. Vol. 38: 450- 457.

Creswell J. W and Creswell J. D (2017). Research Design, Qualitative, Quantitative, and Mixed Methods Approaches, 5th edition, SAGE Publications, Inc. USA

Creswell, J. (2017). Research design: Qualitative, quantitative, and mixed methods approaches .5th ed. Thousand Oaks, CA: SAGE publications 
Department of Health (NDOH (2007). The National Infection Prevention and Control Policy and Strategy. Republic of South Africa. National Department of Health.

Department of Health (NDoH (2011). National Core Standards in Health Establishment in South Africa. Department of Health. Republic of South Africa

Efstathiou, G.; Papastavrou, E.; Raftopoulos, V. and Merkouris, A. (2011). Factors influencing nurses' compliance with Standard Precautions in order to avoid occupational exposure to microorganisms: A focus group study. BMC Nursing. Vol.10:1

Gammon J; Hunt J; Williams S; Daniel S; Rees S; and Matthewson S. (2019): Infection prevention control and organisational patient safety culture within the context of isolation study protocol. BMC Health Serv Res.19(1):296

Ider, E.; Adams, J.; Marton, A.; Whitby, M. and Clement, A. (2012). Perceptions of health care professionals regarding the main challenges and barriers to effective hospitals infection control in Mongolia: a qualitative study. Biomed Central Infections Disease. Vol. 12: 170. Available on: http://www.biomedcentral.com/1471- 2334/12/170.

Lewinsky, L. (2011). Origins of infection prevention research, Florence Nightingale's influence. Dallas. USA: Health Care Art Consulting

Vukoja M; Riviello E.D and Schultz M.J (2018). Critical care outcomes in resource limited settings. Curr Opin Crit Care. Vol. 24(5):421-427. 